\title{
Gesundheitswesen im Umbruch - Brennpunkt Tarife
}

Kerstin Schutz, Susanne Christen

Ressort Ambulante Tarife und Verträge Schweiz
Am 23. Oktober 2013 durfte das Ressort Ambulante Tarife und Verträge Schweiz der FMH fast 100 engagierte Tarifdelegierte und Gäste in Bern begrüssen.

«Gesundheitswesen im Umbruch - Brennpunkt Tarife» lautete das Leitthema der Veranstaltung. Diskutiert wurde unter anderem über den bevorstehenden Eingriff des Bundesrates in die TARMED-Tarifstruktur. Der erste Referent, Dr. med. Ignazio Cassis, Nationalrat und Präsident von «curafutura», eröffnet sein Referat mit einem klaren Statement: Die Tarifpartnerschaft ist kein Auslaufmodell!

Im Anschluss informierten Dr. med. Jürg Schlup, Präsident der FMH, und Dr. med. Ernst Gähler über die aktuelle Situation im ambulanten Tarifbereich.

Am Nachmittag berichteten $\mathrm{FMH}, \mathrm{H}+$ und MTK von den gemeinsamen TARMED-Revisionsarbeiten. Die ersten Fachteams können bald abgeschlossen werden, und neue Fachteams nehmen ihre Arbeiten auf. Ziel ist es, die Revision auf tariftechnischer Ebene bis Ende 2015 abzuschliessen.

Neben Vorträgen im Plenum wurden drei Workshops angeboten:

- Im Workshop «Die Ombudsstelle» gaben lic. iur., RA Morena Hostettler Socha, Ombudsfrau der Krankenversicherung, und Dr. med. Philippe Luchsinger, Ombudsmann der Ärztegesellschaft des Kantons Zürich, interessante Einblicke in ihre Tätigkeiten. Ihre Dienstleistungen tragen dazu bei, die Anzahl von Rechtsverfahren zu verringern, indem Missverständnisse zwischen Krankenkasse und versicherter Person oder zwischen Arzt und Patient durch Aufklärung und Information geklärt werden können.

- Dr. Philip Moline und Dr. Renato Laffranchi von Newlndex zeigten in ihrem Workshop den Prozess der Datenverarbeitung in der ärzteeigenen Datensammlung auf und klärten folgende Fragen: Wie wird der Datenschutz gewährleistet, und warum ist die Datenlieferung an ein Trustcenter wichtig für alle Ärzte? Können Daten darüber Auskunft geben, welche Auswirkungen eine vermehrte Abrechnung im Tiers payant auf die Taxpunktwerte hat?

- Der Workshop für neue Tarifdelegierte wurde zum ersten Mal durchgeführt. Thomas Kessler, Mitarbeiter des Ressorts Ambulante Tarife und Verträge der FMH, erklärte den Teilnehmern, wo relevante Informationen zu den ambulanten Tarifen zu finden sind, wie die Ärztliche Leistung im Modell berechnet wird, welche Parameter eine Tarifposition bilden, und was eigentlich dahintersteckt.

Weiterführende Informationen sowie alle verfügbaren Folien zu den Referaten und Workshops auf der Webseite der FMH.

(www.fmh.ch $\rightarrow$ Ambulante Tarife $\rightarrow$ Publikationen $\rightarrow$ Tarifdelegierten-Tag) 

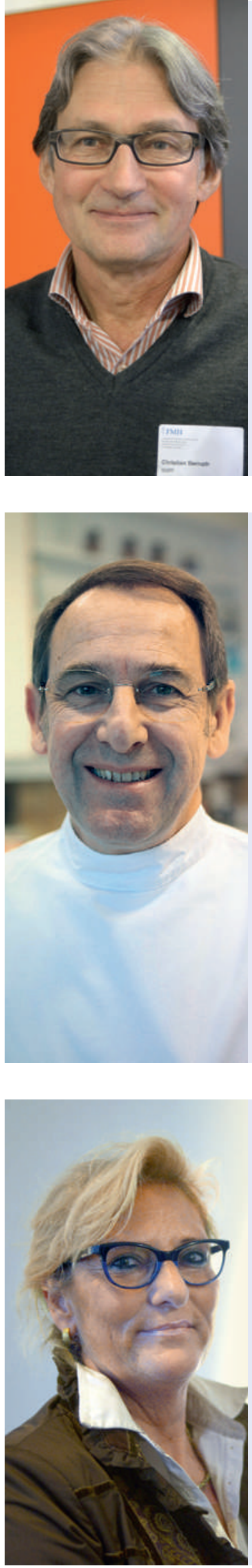

\section{Dr. med. Karin Julia Stadlin, Präsidentin der Ärztegesellschaft Zug}

Was muss die Neuroganisation TARMED Suisse leisten?

Im Vordergrund steht die Tarifautonomie! Das bedeutet Einigkeit zwischen den Verhandlungspartnern. Andernfalls bestimmt der Bundesrat, was einer «Staatsmedizin auf Raten» entspricht! Wichtigste Änderung bei TARMED Suisse muss sein, dass zukünftig Vorlagen im Mehrheitsrecht anstelle der bisherigen «Einstimmigkeit» entschieden werden können.

Dazu kommt ein Vetorecht. Also «Mehrstimmigkeit mit Vetorecht».

Bei der Zusammenarbeit zwischen FMH/ santesuisse/ MTK/ H+ und GDK sollten Tarifstrukturen und nicht Preise im Vordergrund stehen. Die Tarifrevision der FMH sollte von allen Verhandlungspartnern akzeptiert werden.

Ihre Wünsche und Inputs zum Tarifdelegierten-Tag:

Vom Tarifdelegierten-Tag erwarte ich einen Mix aus der aktuellen politischen Situation und Zukunftsvisionen (Bsp. Ärztetarife im Jahre 2020?). 

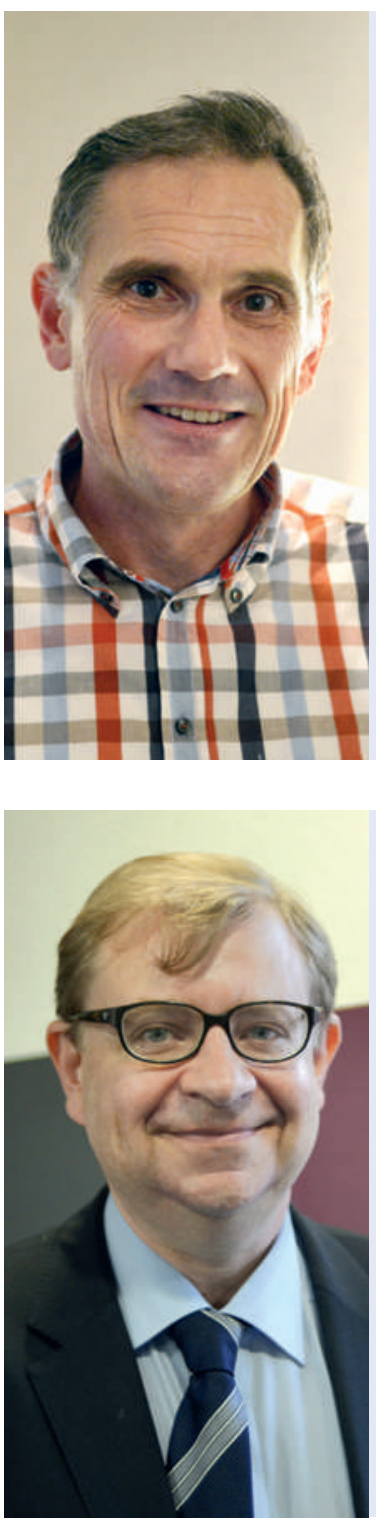

Dr. med. Martin Tönz, Tarifdelegierter der Schweizerischen Gesellschaft für Kinderchirurgie

Was erwarten Sie von einer Gesamtrevision des TARMED?

Das Ziel des TARMED war und ist es, eine korrekte Abgeltung der ärztlichen Leistung zu ermöglichen. Die aktuelle Version des TARMED hat dieses Ziel jedoch nur bedingt erreicht, auf verschiedenen Gebieten ist eine korrekte Abgeltung nicht möglich, so z.B. auf dem Gebiet der Kinderchirurgie. Weiter basiert der TARMED auf betriebswirtschaftlichen Daten, welche nicht mehr der Realität entsprechen. Diese Punkte müssen in der neuen Version berücksichtigt werden.

Die Dignitäten sind eines der «heissesten Eisen» im TARMED. Die Veränderung dieses Parameters wird als mögliche politische Massnahme zur Umverteilung zugunsten der Grundversorger gehandelt. Ist dies aus Ihrer Sicht eine zielführende Massnahme?

Nein, auf keinen Fall. Das führt zu einer Verfälschung des Tarifwerks, so dass es unbrauchbar wird. Dann können wir uns gleich beim Staat für einen fixen Stundenansatz anstellen lassen. Mit einer Staatsmedizin ist dem Grundversorger aber überhaupt nicht geholfen.

Wie sehen Sie die Zusammenarbeit zwischen Ihrer Fachgesellschaft und dem Ressort Ambulante Tarife und Verträge FMH - welche Erwartungen haben Sie?

Ich sehe die Zusammenarbeit in einem konstruktiven Dialog, um mit Hilfe der Fachkenntnisse beider Seiten die komplexe Aufgabe der korrekten Abgeltung der kinderchirurgischen Leistungen zu bewältigen.

\section{Dr. med. Markus Trutmann, Generalsekretär fmch}

Die Dignitäten sind eines der «heissesten Eisen» im TARMED. Die Veränderung dieses Parameters wird als mögliche politische Massnahme zur Umverteilung zugunsten der Grundversorger gehandelt. Ist dies aus Ihrer Sicht eine zielführende Massnahme?

Der TARMED ist für Umverteilungsübungen nicht gemacht worden. Die Dignitäten sind zu belassen oder ganz aufzuheben. Lineare Kürzungen wären nicht statthaft, ja stünden geradezu im Widerspruch zum KVG.

Der neue Player «curafutura» - Segen oder Fluch im Dschungel der Organisationen?

«Curafutura» ist ein Glücksfall. Ihre Charta ist echt liberal. Noch wichtiger ist, dass es neben tarifsuisse auch die HSK als neue Einkaufsgemeinschaft gibt. Der fehlende Wettbewerb beim Leistungseinkauf war einer der grössten Missstände in der Krankenkassenbranche. Jetzt kommt endlich etwas Bewegung ins Spiel.

Ihre Prognose in fünf Jahren: Staatstarife oder Tarifautonomie?

Die Zeichen stehen auf Verstaatlichung unseres Gesundheitswesens. Da werden die Tarife nicht ausgenommen. Für unternehmerisch denkende Ärzte, Spitäler und Versicherungen heisst das: es sind neue Optionen ausserhalb des KVG zu suchen. Lieber heute als morgen.

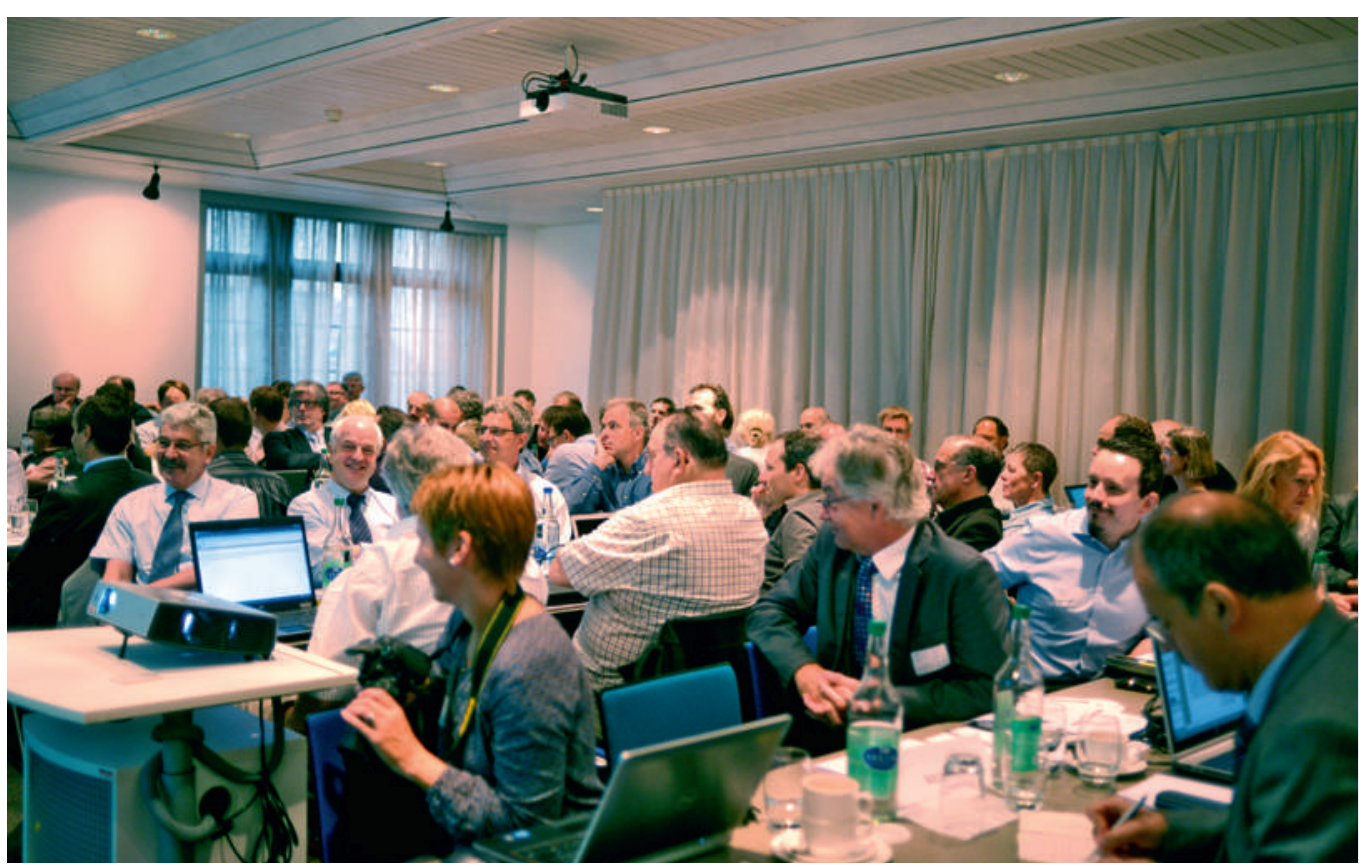

Herzlichen Dank an alle Delegierten für lhre Teilnahme und die konstruktiven Diskussionen.

Jetzt vormerken: nächster Tarifdelegierten-Tag am Mittwoch, 2. April 2014, Hotel Ador, Bern

Kontakt:

FMH

Ambulante Tarife und

Verträge Schweiz

Frohburgstrasse 15

CH-4600 Olten

tarife.ambulant[at]fmh.ch 